\title{
Episodic Memory Retrieval Functionally Relies on Very Rapid Reactivation of Sensory Information
}

\author{
Gerd T. Waldhauser, ${ }^{1,2}$ Verena Braun, ${ }^{1,3}$ and Simon Hanslmayr ${ }^{1,3}$ \\ ${ }^{1}$ Department of Psychology, University of Konstanz, 78457 Konstanz, Germany, ${ }^{2}$ Department of Neuropsychology, Institute of Cognitive Neuroscience, \\ Ruhr-University Bochum, 44801 Bochum, Germany, and ${ }^{3}$ School of Psychology, University of Birmingham, Edgbaston, B15 2TT, Birmingham, United \\ Kingdom
}

Episodic memory retrieval is assumed to rely on the rapid reactivation of sensory information that was present during encoding, a process termed "ecphory." We investigated the functional relevance of this scarcely understood process in two experiments in human participants. We presented stimuli to the left or right of fixation at encoding, followed by an episodic memory test with centrally presented retrieval cues. This allowed us to track the reactivation of lateralized sensory memory traces during retrieval. Successful episodic retrieval led to a very early $(\sim 100-200 \mathrm{~ms})$ reactivation of lateralized alpha/beta $(10-25 \mathrm{~Hz})$ electroencephalographic (EEG) power decreases in the visual cortex contralateral to the visual field at encoding. Applying rhythmic transcranial magnetic stimulation to interfere with early retrieval processing in the visual cortex led to decreased episodic memory performance specifically for items encoded in the visual field contralateral to the site of stimulation. These results demonstrate, for the first time, that episodic memory functionally relies on very rapid reactivation of sensory information.

Key words: ecphory; EEG; episodic memory; oscillations; retrieval; TMS

\section{Significance Statement}

Remembering personal experiences requires a "mental time travel" to revisit sensory information perceived in the past. This process is typically described as a controlled, relatively slow process. However, by using electroencephalography to measure neural activity with a high time resolution, we show that such episodic retrieval entails a very rapid reactivation of sensory brain areas. Using transcranial magnetic stimulation to alter brain function during retrieval revealed that this early sensory reactivation is causally relevant for conscious remembering. These results give first neural evidence for a functional, preconscious component of episodic remembering. This provides new insight into the nature of human memory and may help in the understanding of psychiatric conditions that involve the automatic intrusion of unwanted memories.

\section{Introduction}

Perceived information can reverberate with stored memory traces (Tulving et al., 1983). This fast and involuntary process is considered a decisive ingredient for the "mental time travel" implied in episodic memory retrieval, reinstating sensory features of study episodes and biasing subsequent retrieval processes leading

Received June 1, 2015; revised Nov. 17, 2015; accepted Nov. 23, 2015.

Author contributions: G.T.W., V.B., and S.H. designed research;G.T.W. and V.B. performed research;G.T.W., V.B., and S.H. analyzed data; G.T.W., V.B., and S.H. wrote the paper.

This work was supported by the Swedish Research Council (Grant VR 435-2011-7163) and the University of Konstanz Young Scholar Fund (Grant 83946931) to G.T.W., and the German Research Council (Grant DFG HA 5622/ 1-1) to S.H. We thank Martin Dahl for valuable help during data collection and Tzvetan Popov for assistance with EEG source analysis.

The authors declare no competing financial interests.

Correspondence should be addressed to Dr. Gerd T.Waldhauser, Department of Neuropsychology, Institute of Cognitive Neuroscience, Ruhr-University Bochum, GAF0 05/602, Universitätsstrasse 150, 44801 Bochum, Germany. E-mail: gerd.waldhauser@rub.de.

DOI:10.1523/JNEUROSCI.2101-15.2016

Copyright $\odot 2016$ the authors $\quad 0270-6474 / 16 / 360251-10 \$ 15.00 / 0$ to the experience of recollection (Tulving, 1983; Tulving et al., 1983). This fundamental memory mechanism, termed "ecphory," has long been described phenomenologically and studied behaviorally (Semon, 1911; Tulving, 1976; 1982). Neurophysiological evidence for such fast reinstatement processes has not emerged until recently (Waldhauser et al., 2012; Wimber et al., 2012; Jafarpour et al., 2014). Using electrophysiological methods, these recent studies show that a reactivation of sensory memory traces can occur very rapidly, within $500 \mathrm{~ms}$ after onset of a retrieval cue. However, whether this early reactivation process functionally drives episodic memory retrieval, as assumed in the theoretical idea of ecphory, remains unclear. We used a visualhalf field paradigm to identify the oscillatory signature of early reactivation (Experiment 1) and, moreover, to show that ecphoric reactivation is functionally relevant for episodic memory retrieval (Experiment 2).

Retrieval from episodic memory leads to a reactivation of sensory memory traces in the hemisphere contralateral to the visual 


\section{Procedure Experiments 1 and 2}
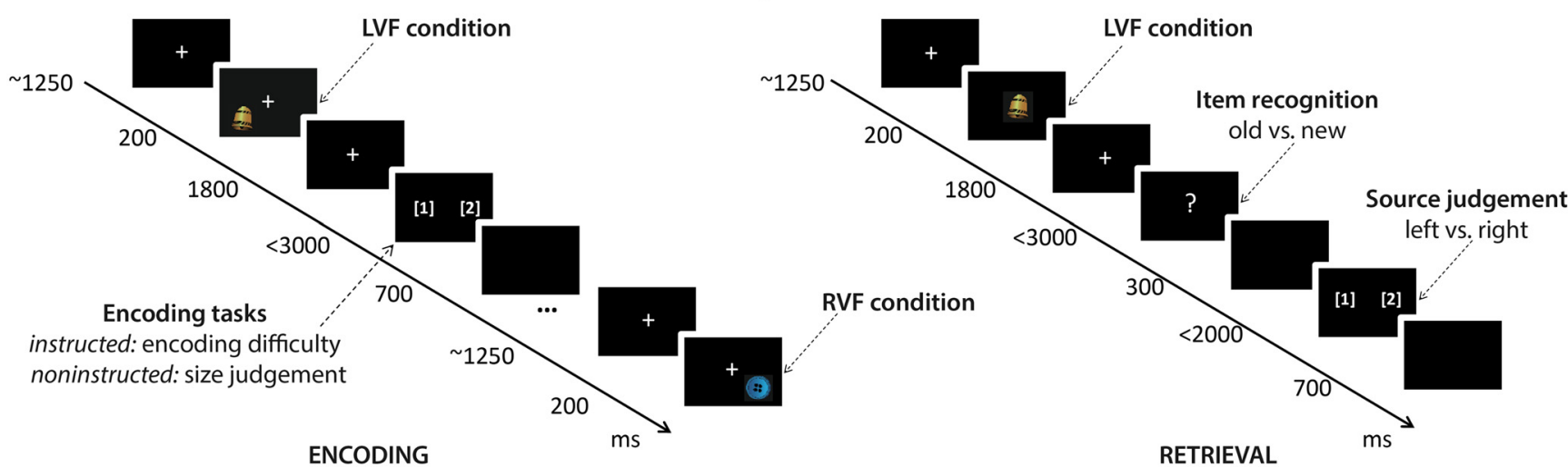

Figure 1. Experimental procedure for Experiments 1 and 2. Pictures of everyday objects were presented to the left or right of fixation at encoding, followed by a response task according to encoding condition (instructed vs noninstructed). Instructed encoding required participants to intentionally encode the presented object and to judge the difficulty to do so. For noninstructed encoding, participants were requested to estimate whether or not the depicted object would fit into a shoebox. During retrieval, all previously shown old items were presented together with the same amount of previously unseen new items. All pictures were shown at the center of the screen to isolate lateralized cortical activity to the reactivation of sensory memory traces established during encoding. An old/new item recognition task was followed by a source memory task, asking for the VF at presentation. The whole procedure was performed twice in each subject: once with instructed, once with noninstructed encoding. In Experiment 1, EEG was measured throughout the experiment. In Experiment 2, rTMS and Sham was applied to the left or right cortical hemisphere during retrieval, switching between these stimulation conditions every 40 trials.

field of initial presentation (Slotnick and Schacter, 2006; Waldhauser et al., 2012). We made use of this principle to track the rapid reactivation of individual memory representations, measuring and modulating brain oscillatory activity by means of electroencephalography (EEG, Experiment 1) and rhythmic transcranial magnetic stimulation (rTMS, Experiment 2). Participants were engaged in instructed or noninstructed encoding of everyday objects presented either in the left or right visual field (LVF/RVF; see Fig. 1). In a subsequent retrieval task, memory cues were presented at the center of the screen and participants engaged in item recognition followed by a source memory task. Source memory was examined to test for the ability to retrieve contextual details of the study episode, which is considered a hallmark of episodic memory (Tulving, 1983).

Previous studies were able to localize memory reactivation effects during retrieval to lateralized visual cortical areas active during encoding (Gratton, 1998; Slotnick, 2004; Slotnick and Schacter, 2006) and investigated the timing of lateral reactivation in terms of event-related potential effects (Gratton, 1998; Slotnick and Schacter, 2010). However, it still remains unclear whether and how rapid visual cortical reactivation is causally relevant to explicit episodic memory (Slotnick and Schacter, 2010; Thakral et al., 2013), leaving a fundamental question of memory theory unanswered. To shed light on these questions, we investigated brain oscillations as a highly sensitive, physiologically relevant measure of cortical activity. Following recent ideas (Hanslmayr et al., 2012), a desynchronization of oscillatory power in the $\alpha / \beta$ frequency bands indicates an increase in the complexity of firing patterns, thereby allowing for higher information coding capacity during memory encoding and retrieval. Thus, we expected a specific decrease of $\alpha / \beta$ oscillations in the brain hemisphere contralateral to the visual field of encoding as a marker of memory reactivation. In line with the theoretical notion of ecphory, this $\alpha / \beta$ power decrease was hypothesized to occur very rapidly (i.e., within $500 \mathrm{~ms}$ ) (Tulving et al., 1983; Waldhauser et al., 2012; Jafarpour et al., 2014), before the occurrence of signatures of recollection and controlled retrieval processes (Rugg and Curran, 2007). Applying rTMS at the neural sources, in the time range, and with a frequency as identified in the first experiment was expected to counteract the rapid reacti- vation of visual cortical areas and lead to disrupt source memory performance. This would give strong evidence for the functional relevance of ecphoric processes for episodic remembering.

\section{Materials and Methods}

Materials and procedure

Materials, behavioral task, trial timing, and basic procedure were identical for Experiments 1 and 2 (see Fig. 1). The study was approved by the Ethical Review Board at the University of Konstanz.

Stimulus material. A total of 240 everyday objects (Rossion and Pourtois, 2004) were used in each experiment. Half of these items were presented during encoding, serving as old items during retrieval. Objects subtended a visual angle of $5.6^{\circ} \times 4^{\circ}$ and were presented to the left or right visual field (LVF/RVF), $4^{\circ}$ below and $6^{\circ}$ lateral from central fixation. Items were preselected into 8 sets, so that semantic categories of the depicted objects, frequency of occurrence, vividness, visibility, and orientation (portrait vs landscape) were balanced across conditions.

Procedure. The experiment was performed in two blocks, pertaining to the two encoding conditions (instructed and noninstructed encoding). Each block comprised the encoding phase, a distracter task, and a final retrieval phase. In the noninstructed encoding condition, participants were supposed to engage in judging the size of each object. In the intentional encoding condition, subjects were simply instructed to intentionally encode each object the best they could for later retrieval. During encoding, participants responded with the index and middle finger of the right hand whether an item was larger or smaller than a shoebox or whether the item was easy or difficult to encode. Allocation of response buttons to index or middle finger was counterbalanced across subjects. The encoding phase was followed by a 3 min distracter task to prevent selective rehearsal, consisting of counting backwards aloud from a three digit number in steps of three. During the subsequent retrieval task, 60 encoded items were presented at the center of the screen as old items together with the same amount of new items. Participants were instructed to first engage in an old/new recognition test and subsequently tested for source memory, indicating whether an item was presented to the left or right visual field during encoding. Participants responded with the index and middle finger of the right hand whether an item was old or new and whether the item was endorsed as old, whether the item was initially presented to right or left of fixation. Allocation of response buttons to index or middle finger was counterbalanced across subjects in the old/new task, but the middle finger was always used to indicate source endorsement to the RVF and the index finger was used to indicate LVF presentation. Succession of encoding conditions, old/new status of items, 
and presentation of the old items to the left or right visual field during encoding were counterbalanced across subjects.

Presentation of items within encoding phases was randomized so that each VF condition was followed by an item from the same or opposite VF condition with equal probability and under the constraint that the same VF could occur twice in a row. During retrieval, the sequence of items encoded in the left and right VF was also randomized under the same constraint (no more than two items from the same VF condition in a row). Additionally, old items were randomly intermixed with new items so that no more than four old or new items could occur in a row.

Analysis of behavioral data. Behavioral data during the final memory test were analyzed based on (1) hits (i.e., the number of correctly identified old items) and (2) source hits (i.e., the number of hits for which VF of presentation was correctly identified in addition to correct item recognition). Number of hits and source hits were each divided by the number of all old items for each subject. Arguably, dividing the number of source hits by the number of hits would give a cleaner measure of pure source memory performance in addition to simple item recognition. However, we assumed that source memory performance is already constrained by item memory performance in our case, so that a "new" response to an old item (i.e., miss) prevents a correct source memory judgment, as only an "old" response prompts a subsequent source memory task. In addition, item recognition performance may already be biased by source memory demands so that only items are endorsed as hits if a correct source memory judgment can be made. As a consequence of our calculation method, source hit rates may appear rather low. Chance level for source hits is $<50 \%$ because the probability to endorse an item as being presented in the LVF or RVF already depends on the probability to endorse an item as old or new (for detailed discussion, see, e.g., Cycowicz et al., 2001; Batchelder and Riefer, 1990). In addition to the different types of hits, we report false alarms (new items that were incorrectly classified as old). For Experiment 2, we also investigated source false alarms (new items that were incorrectly endorsed as old and for which a source memory judgment was given). This analysis was conducted to determine whether rTMS affects the tendency to misattribute a new item to the VF contralateral or ipsilateral of stimulation. Behavioral data were analyzed in uncorrected repeated-measures ANOVAs or uncorrected two-sided paired $t$ tests.

\section{Experiment 1}

Participants. Sixteen right-handed (Oldfield, 1971) subjects (11 female) with a mean age of 24 years (range 21-37 years), normal or corrected-tonormal vision, and no history of neurological of psychiatric disease participated in this study. Participants received course credit or a monetary reward of $20 €$ for participating in the experiment. Informed consent was acquired from each participant before the experiment.

Data analysis. EEG was recorded using equidistant $128 \mathrm{Ag} / \mathrm{AgCl}$ scalp electrodes (DC-100 Hz, $512 \mathrm{~Hz}$ sampling rate) referenced to common average. A time-frequency representation of the EEG signal $(1-30 \mathrm{~Hz})$ was derived by means of Morlet wavelets (width 5). Because we had no hypotheses concerning the different encoding tasks and because no statistical differences between encoding tasks were obtained (see Results; Table 1), EEG power was collapsed across encoding tasks. To quantify event-related signal changes on sensor level, poststimulus power change was calculated in relation to a prestimulus baseline period ( -500 to $0 \mathrm{~ms}$ ) for both encoding and retrieval phases. No significant electrode clusters emerged between conditions in the baseline interval in a fieldtrip cluster statistic calculated at all electrodes for the frequency ranges of interest during encoding and retrieval.

To identify time windows and the frequency range of interest, EEG power during encoding was first compared between LVF and RVF conditions in a sliding-time window fieldtrip cluster statistic (Maris and Oostenveld, 2007). Here, we continuously applied cluster statistics for time and frequency windows of $200 \mathrm{~ms}$ and $2 \mathrm{~Hz}$ in steps of $100 \mathrm{~ms}$ and $1 \mathrm{~Hz}$ to the data from 1 to $30 \mathrm{~Hz}$ and from 0 to $1500 \mathrm{~ms}$ (Staudigl and Hanslmayr, 2013). Following our hypotheses, we directly compared posterior electrodes over the respective hemisphere contralateral to the VF of presentation between LVF and RVF conditions. We did so by inverting EEG power values in the RVF condition for each time and frequency point at left-hemispheric parieto-occipital sensors through multiplication with -1 . Then, we compared the result in each time-frequency window with the corresponding (noninverted) power values at righthemispheric sensors for the LVF condition. Each cluster statistic then reveals whether there is a contralateral power decrease, with the highest likelihood of significant effects if there is a strong contralateral decrease in both LVF and RVF conditions. To correct for multiple comparisons, the resulting $630 p$ values were adjusted following the false discovery rate procedure (Benjamini and Hochberg, 1995; Benjamini and Yekutieli, 2001). The result of this analysis indicated electrode clusters yielding significant EEG power differences between LVF and RVF conditions over the respective contralateral hemispheres in the alpha/ lower beta range $(8-20 \mathrm{~Hz})$, most prominent between 200 and $700 \mathrm{~ms}$ after stimulus presentation (see Fig. $3 A$, top).

However, the sliding cluster analyses can only suggest the presence of a statistical difference between VF conditions at the contralateral hemispheres, but it is not able to reliably show the actual topographical clusters that differ between VF conditions and to take into account ipsilateral and contralateral sensors. To this end, we subtracted noninverted data in the RVF from the LVF condition and calculated a fieldtrip cluster statistic in the time ( $200-700 \mathrm{~ms})$ and frequency $(8-20 \mathrm{~Hz})$ window suggested by the sliding analysis, allowing us to identify significant electrode clusters in each hemisphere (see Fig. 3A, middle). The mean EEG power at these clusters interacted significantly between $\mathrm{VF}(\mathrm{LVF} / \mathrm{RVF})$ and left and right brain hemispheres (LH/RH; see Fig. $3 A$, bottom), as indicated by a twoway ANOVA.

To investigate lateralized activity during retrieval, EEG power differences between contralateral and ipsilateral electrode clusters identified during encoding were averaged over LVF and RVF conditions and compared over time in a frequency range between 1 and $30 \mathrm{~Hz}$. Results were masked by the results of running Wilcoxon signed-rank test applied at each time $(\sim 50 \mathrm{~ms})$ and frequency $(\sim 0.5 \mathrm{~Hz})$ bin resulting from the Morlet wavelet time-frequency transformation. Because our hypotheses were concerned with rapid contralateral decreases in $\alpha / \beta$ power, we focused on early $(<500 \mathrm{~ms})$ interaction effects as indicators for ecphoric processes. Negative values in Figure $3 B$ (top) indicate uncorrected significant $(p<0.05)$ differences between contralateral - ipsilateral electrodes collapsed across both VF conditions. Based on the results of this exploratory analysis, the interaction between VF (LVF/RVF) and left and right brain hemispheres was tested in an uncorrected two-way repeatedmeasures ANOVA taking into account mean EEG power $(10-25 \mathrm{~Hz}$, $100-200 \mathrm{~ms}$ ) at the LH/RH clusters identified during encoding (see Fig. $3 B$, bottom, for mean power depending on condition and hemisphere and Fig. $3 B$, middle, for topographical distribution of mean EEG power). To shed light on hemisphere specificity of these effects (see Fig. 4), additional two-sided $t$ tests were performed in the 100-200 ms time window, comparing LVF-RVF differences in the hemisphere-specific peak frequencies at the $\mathrm{LH}$ and $\mathrm{RH}$ electrode clusters.

To test whether reinstatement strength reflecting in a contralateral $\alpha / \beta$ power decrease is linked to behavioral performance, we compared the amount of lateralization between source hits and source misses in a twosided $t$ test. Lateralization was defined as the mean difference between the contralateral - ipsilateral electrode clusters in the $10-25 \mathrm{~Hz}$ frequency band from 100 to $200 \mathrm{~ms}$. We expected lateralization to be more negative for source hits than source misses. In a second step, we also investigated whether single trials could be identified as source hits and source misses (i.e., hits that were incorrectly attributed to the VF opposite to the VF of actual presentation) based on the amount of contralateral - ipsilateral lateralization at the electrode clusters in the $10-25 \mathrm{~Hz}$ frequency band by means of a contingency table (Table 2). In this approach, EEG signals were not baseline corrected to avoid misclassification of trials due to potentially higher baseline noise in nonaveraged single trials. Instead, a lateralization index was estimated for each trial by correcting for the total power at the contralateral and ipsilateral electrodes (lateralization in$\operatorname{dex}=$ contralateral - ipsilateral/contralateral + ipsilateral) (compare Händel et al., 2011). A lateralization index $<0$ signifies a trial as having a relative power decrease at contralateral electrodes. We hypothesized that such trials would be classified as source hits with a frequency above chance, whereas trials with a lateralization index $>0$ would more often be 
Table 1. Memory performance depending on encoding condition in Experiments 1 and $2^{a}$

\begin{tabular}{|c|c|c|c|c|c|c|c|c|}
\hline & Experiment 1 & & & & Experiment 2 & & & \\
\hline & Instructed & Noninstructed & LVF & RVF & Instructed & Noninstructed & LVF & RVF \\
\hline Hits & $67.9 \pm 3.35$ & $71.3 \pm 1.45$ & $71.2 \pm 2.25$ & $68.0 \pm 2.76$ & $69.7 \pm 2.48$ & $72.0 \pm 2.81$ & $69.4 \pm 2.71$ & $73.3 \pm 2.51$ \\
\hline Source hits & $55.7 \pm 4.01$ & $50.3 \pm 2.31$ & $53.1 \pm 2.95$ & $52.9 \pm 2.96$ & $56.8 \pm 2.90$ & $52.0 \pm 3.03$ & $54.0 \pm 2.85$ & $55.9 \pm 2.91$ \\
\hline
\end{tabular}

${ }^{a}$ Percentages of hits and source hits (mean \pm SEM) did not differ between VF (LVF and RVF) or instruction (Instructed vs Noninstructed) at encoding (see Results).

classified as source misses. We tested this assumption by means of a $\chi^{2}$ test for all trials from all participants (i.e., fixed-effects analysis). To make the data more accessible, we also report classification performance in percentage ((correctly classified source hits + correctly classified source misses)/all items) $\times 100$ ). In addition, we also tested whether the mean differences between observed cell frequencies and expected cell frequencies in $\chi^{2}$ tests across all participants were significantly different from zero on the group level by means of a one-sample $t$ test (i.e., randomeffects analysis).

To identify sources of oscillatory activity, we used the Dynamic Imaging of Coherent Sources beam-forming approach after calculating the cross-spectral density matrix. We used individual electrode positions as acquired with a sensor digitization tool (Xensor; www.ant-neuro.com) and the FieldTrip standard MRI. Dynamic Imaging of Coherent Sources constructs adaptive spatial filters to localize power for each grid point in the entire brain (Gross et al., 2001; Gross et al., 2003). For each individual and the time periods of interest during encoding and retrieval, filters were calculated using activity following the encoding stimulus and the recognition cue, respectively, and baseline periods corresponding to the length of the poststimulus period of interest (Dalal et al., 2008), including the trials from both LVF and RVF conditions. For encoding data, a baseline period from -500 to $0 \mathrm{~ms}$ and poststimulus period from 200 to 700 $\mathrm{ms}$ were chosen. For retrieval data, a baseline period of -100 to $0 \mathrm{~ms}$ and a poststimulus period of 100 to $200 \mathrm{~ms}$ were chosen. Cross-spectral density and source power were estimated using frequency analysis with Slepian multitapers as implemented in FieldTrip (Oostenveld et al., 2011) for the frequency range observed at sensor level (i.e., $8-20 \mathrm{~Hz}$ for encoding and $10-25 \mathrm{~Hz}$ for retrieval). The resulting average source estimate for each condition in the time intervals of interest was corrected for source activity in the baseline interval and subsequently statistically compared with the other condition and interpolated to the standard MNI brain. In a final step, we aimed at identifying the voxels that differentiated with most sensitivity between conditions across both hemispheres. To this end, we statistically compared the RVF-LVF power difference in the right hemisphere with the corresponding effect in the left hemisphere in a one-sided FieldTrip source cluster statistic. This was done again for both encoding and retrieval effects. The results were again interpolated to the standard MNI brain (see Fig. 5). The obtained maximum interhemispheric difference between conditions during retrieval was determined at MNI coordinates $\pm 40,-78,0$. Source localization results were visualized using the Caret software (http://brainvis.wustl.edu).

\section{Experiment 2}

Participants. Twenty-four right-handed subjects ( 14 female) with a mean age of 23 years (range 18-27 years) and normal or corrected-to-normal vision completed the experiment. In addition to a general assessment of history of neurological and psychiatric disease and medication, an additional TMS screening was conducted before the experiment (Rossi et al., 2011). Furthermore, a detailed explanation of the TMS method and its risks was provided for the subjects (Rossi et al., 2009). Informed consent was acquired from each participant before the experiment. One participant was excluded before the start of the study due to unclear status of inner ear damage, and one further person aborted the experiment because of neck pain during phosphene stimulation. Participants received course credit or a monetary reward of $20 €$ for participating in the experiment and an extra $10 €$ for taking part in structural MRI assessment.

Procedure. Behavioral task and procedure were almost identical to Experiment 1. Stimulation conditions were applied in a within-subject fashion during the retrieval phase of both blocks. This required pausing the procedure every 40 trials to relocate the coil between the hemispheres and change the tilt of the coil according to TMS and sham conditions.
Old, new, LVF, and RVF items were equally distributed between these segments of 40 trials. Four subjects received only 36 trials per stimulation block due to experimental error, but counterbalancing and matching of condition and stimulus material were preserved. rTMS was applied to the right and left hemisphere during each of the two retrieval phases. Sham was applied to only one hemisphere during a particular retrieval block to keep the blocks at reasonable size and length. Succession of rTMS and Sham conditions was counterbalanced across subjects so that stimulation conditions and hemispheres were equally often followed and preceded by the other conditions.

TMS was applied with a Magstim Rapid ${ }^{2}$ Transcranial Magnetic Stimulator via a Magstim figure-of-eight coil. At the beginning of an experimental session, phosphene threshold (PT) was identified in each subject, using the modified binary search procedure (Tyrrell and Owens, 1988; Anderson and Johnson, 2006; Romei et al., 2010). To this end, subjects were blindfolded and single TMS pulses were applied with the coil centered midline $2 \mathrm{~cm}$ above the inion. Participants had to indicate the presence or absence of a phosphene by answering "yes" or "no." PT is defined as the percentage of maximum stimulator output above which subjects consistently report seeing phosphenes. PT served as an individual marker of cortical excitability and was used to determine stimulator output during the experiment. rTMS was applied with 90\% PT output, which corresponded to a mean stimulator output of $61.3 \%$ (SD 7.44\%). If no reliable PT could be determined $(n=3)$, a fixed stimulator output of $60 \%$ was used (Romei et al., 2010). No participant reported perception of phosphenes during the experiment.

To deliver magnetic pulses with high anatomical precision, rTMS was guided by a neuronavigation system, which coregisters the individual MRI with the position of the TMS coil using a 3D tracking device (ANTVisor; www.ant-neuro.com). Individual high-resolution T1weighted MRIs were acquired from a Siemens Skyra $3 \mathrm{~T}$ scanner (flip angle $=7^{\circ} ; \mathrm{TR}=2500 \mathrm{~ms}$; TE $=4.06 \mathrm{~ms}$ ) or based on other structural MRI scans from various sources if already existing for the participant. Maximum magnetic field strength was applied to the neural source of $\alpha / \beta$ oscillatory decreases in the lateral occipital cortex as identified during retrieval in Experiment 1 (MNI coordinates: $\pm 40,-78,0$ ). Four TMS pulses were applied with a duration of $57 \mathrm{~ms}$ between pulses, with application starting at $33.5 \mathrm{~ms}$ and lasting until $204.5 \mathrm{~ms}$ after cue presentation, corresponding to a frequency of $17.5 \mathrm{~Hz}$ (see Fig. 6A).

Sham was applied by tilting the TMS coil in an angle of $\sim 60^{\circ}$ away from the scalp surface but still touching the head. Thereby, participants still experienced clicking noise and slight vibrating sensation at the scalp surface (Sauseng et al., 2009).

\section{Results}

Episodic memory retrieval is accompanied by a rapid decrease of $\boldsymbol{\alpha} / \boldsymbol{\beta}$ oscillatory power

In Experiment 1 (Fig. 1), high-density EEG was measured during encoding and retrieval to reveal the oscillatory signature of memory reactivation. In a first step, we aimed at identifying encodingrelated lateralized brain activity. On a behavioral level, no difference between encoding tasks (instructed vs noninstructed) or visual field conditions (LVF vs RVF) were obtained, on either item recognition or on source memory performance (all $t_{(15)}<$ $1.361, p>0.05$; Table 1). In addition, there was no difference between blocks determined by encoding conditions in terms of false alarm rates $\left(t_{(15)}=1.156, p=0.266\right.$; instructed: mean $\pm \mathrm{SD}$, $11.6 \pm 8.91 \%$; noninstructed: $14.1 \pm 8.65 \%)$. This allowed us to collapse the EEG data across encoding instructions to enhance 


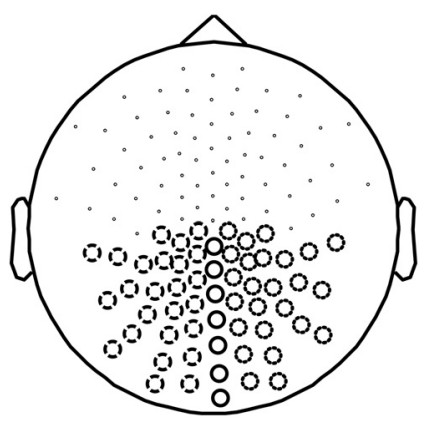

Figure 2. Posterior electrodes selected for analysis at encoding. Lateral electrodes selected for the initial sliding cluster statistic are depicted in large broken (LH) and dotted (RH) circles. In the topographical cluster analysis comparing LVF and RVF conditions, central electrodes (large solid black) were also included.

signal-to-noise ratio and to directly compare VF conditions on a neural level. We compared EEG activity between RVF and LVF conditions at the respective left- or right-hemispheric posterior contralateral electrodes in a sliding cluster statistic (see Materials and Methods; Fig. 2) to isolate the time and frequency windows most sensitive to the expected contralateral power decreases at encoding. This was achieved by inverting power values for the RVF condition at the left-hemispheric electrodes and comparing them with power at right-hemispheric electrodes for the LVF condition. This analysis indicated maximum power differences at contralateral electrodes between the VF conditions from 200$700 \mathrm{~ms}$ in the $\alpha / \beta$ range $\left(8-20 \mathrm{~Hz}, p_{\text {adj }}<0.05\right.$, FDR corrected; Fig. 3A, top) (Genovese et al., 2002). Although this result pinpointed the time and frequency range of lateral differences between VF conditions, the analysis could neither indicate whether the effect is stronger on contralateral than ipsilateral electrodes nor answer exactly which electrodes are most sensitive to hemisphere and VF-specific effects. Thus, we subtracted power in the LVF condition from power in the RVF condition in the identified time-frequency range, such that positive values reflect a stronger $\alpha / \beta$ power decrease in the RVF condition, whereas negative values reflect a power decrease in the LVF condition (Fig. $3 A$, middle). We calculated a cluster statistic on all electrodes to identify the clusters most sensitive to VF specific decreases (Maris and Oostenveld, 2007). In line with our hypothesis, this analysis revealed one left-hemispheric and one right-hemispheric electrode cluster (left: $p=0.038$; right: $p=0.006$ ), each most sensitive to a power decrease in the contralateral VF (Fig. $3 A$, middle). This $\mathrm{VF} \times$ Hemisphere interaction was supplemented by a two-way repeated-measures ANOVA taking into account mean power at the identified electrode clusters $\left(F_{(1,15)}=62.039, p<0.001\right)$, suggesting a significant power decrease at contralateral electrodes differing from ipsilateral activity for each VF condition (see Fig. $3 A$, bottom). Finally, we conducted a beamformer source analysis of the $8-20 \mathrm{~Hz} \alpha / \beta$ oscillatory activity between 200 and $700 \mathrm{~ms}$ (Gross et al., 2001; Gross et al., 2003). To obtain the maximum interhemispheric difference between conditions, we subtracted the interpolated RVF-LVF power difference at source level in the right hemisphere from the effect in the left hemisphere and vice versa. The source analysis, localizing the maximum difference between contralateral and ipsilateral hemispheres, revealed neural generators of the EEG effect in the lateral (middle and inferior) occipital gyrus (LOC, BA 18/19; see Fig. 5).

To reveal the neural signature of memory reactivation, we performed statistical analyses on those electrode clusters showing significant lateralization differences between LVF and RVF at encoding. Following previous EEG studies of memory reactivation (Wimber et al., 2012; Jafarpour et al., 2014; Johnson et al., 2015) and in line with the theoretical notion of ecphory (Tulving et al., 1983), we focused our analysis at retrieval on a time-range preceding the typical timing of recollection effects, before $500 \mathrm{~ms}$ after presentation of the retrieval cue. As indicated by a continuous Wilcoxon sign-rank test, a significant difference between ipsilateral and contralateral electrode clusters emerged very early, $100-200 \mathrm{~ms}$ after cue presentation $(p<0.05)$, and in a similar frequency band as during encoding, between 10 and $25 \mathrm{~Hz}$ (Fig. $3 B$, top). This difference, again, was due to a power decrease at the electrode cluster contralateral to the VF of presentation during encoding, as indicated by a significant VF $\times$ hemisphere interaction $\left(F_{(1,15)}=8.773, p=0.01\right.$, see Fig. $\left.3 B\right)$. This effect appeared to be different between hemispheres in terms of peak frequencies. As shown in Figure $4 A$, differences between VF conditions were clustered $\sim 20 \mathrm{~Hz}$ in the RH cluster, whereas the LH cluster displayed a more specific effect at $\sim 10 \mathrm{~Hz}$. Frequency specificity of the two hemispheres was confirmed in a statistical analysis, showing that only the LH cluster showed a significant VF difference at $10 \mathrm{~Hz}\left(t_{(15)}=2.332, p=0.034\right)$ and only the RH cluster showed a significant VF difference at $20 \mathrm{~Hz}\left(t_{(15)}=2.616, p=0.02\right.$; Fig. $4 B)$. However, based on our previous studies and following the idea that we tap into similar oscillatory processes in the two hemispheres, we continued our analyses with data integrated over the whole frequency range showing a mean contralateral versus ipsilateral difference (Waldhauser et al., 2012).

To check whether contralateral versus ipsilateral differences in the $10-25 \mathrm{~Hz}$ frequency range are indeed due to a poststimulus decrease, and not due to a prestimulus shift of attention, we repeated the same analysis taking into account the raw data from the baseline $(-500$ to $0 \mathrm{~ms})$ interval. This analysis yielded no significant results $\left(F_{(1,15)}<3.554, p>0.05\right)$.

According to theories on episodic memory, an ecphoric reactivation of trace information is a prerequisite for the recollection of details of an episode. Thus, we expected it to be most pronounced for source hits, for which the source of encoding could be correctly remembered. In line with this idea, the contralateral versus ipsilateral $\alpha / \beta$ power decrease was significantly greater for source hits $(-7.13 \pm 9.631 \%)$, compared with source misses $\left(t_{(15)}=4.061, p=0.001\right)$, the latter actually showing a relative power increase at contralateral electrodes $(7.27 \pm 12.534 \%)$. The lateralization effects for source hits and source misses were both significantly different from zero (source hits: $t_{(15)}=-2.962, p=$ 0.01 ; source misses: $\left.t_{(15)}=2.320, p=0.035\right)$. These results suggest that the laterality of EEG power predicts to which VF an item is attributed to, in a way that subjects tend to attribute an item to the VF that is contralateral to the hemisphere displaying a power decrease. As shown in Table 2, this was also the case on a singletrial level. Source hit trials more often showed a negative lateralization index (i.e., a power decrease at electrodes contralateral to the VF in which the item was actually presented during encoding). Source miss trials more often showed a positive lateralization index (i.e., a power decrease over the ipsilateral hemisphere) (Table 2). A dependency of lateralization and response was confirmed statistically in a fixed-effects analysis $\left(\chi_{1}^{2}=4.731, p=\right.$ 0.03 ), showing that $53 \%$ of all items were classified correctly as source hits and source misses based on EEG lateralization. This statistical dependency was also confirmed in a random-effects analysis on group level, with the mean difference between observed and expected cell values being significantly greater than zero $\left(0.951 \pm 1.6374 ; t_{(15)}=2.25, p=0.04\right)$. Notably, 11 (of 16) participants numerically showed this classification pattern (i.e., 
mean differences $>0$ ) on the single-trial level. Lateralization and memory performance were independent when taking into account the baseline interval from -500 to $0 \mathrm{~ms}$ on a single-trial level $\left(\chi_{1}^{2}=\right.$ 1.8467, $p=0.174)$. Classification of source hits and source misses across all subjects based on EEG lateralization was close to chance (49\%). This accorded with a random-effects analysis when comparing individual differences between expected and observed frequencies against zero $\left(0.647 \pm 1.317 ; t_{(15)}=1.885, p=\right.$ $0.079)$.

Finally, we aimed at localizing the neural generators of $\alpha / \beta$ power decreases for source hits. Neural generators of this EEG effect corresponded closely to the sources observed during encoding, localized to the middle and inferior LOC (BA 18/19; Fig. 5).

Together, these data indicate a very rapid reactivation of neural signatures established during encoding, which are visible in a power decrease in the $\alpha / \beta$ frequency band. Source analysis suggests that $\alpha / \beta$ decreases can be localized to the LOC, a region that is known to be constitutive for object recognition during perception and encoding (Konen and Kastner, 2008) and that is sensitive to hemisphere-specific retrieval of lateralized visual memory traces (Slotnick, 2004; Slotnick and Schacter, 2006).

The very early $(\sim 100 \mathrm{~ms})$ reemergence of $\alpha / \beta$ oscillatory decreases during retrieval is in line with previous EEG studies of early reactivation (Wimber et al., 2012; Jafarpour et al., 2014; Johnson et al., 2015) and suggests that retrieval correlates with a rapidly occurring ecphoric process. However, it is unclear whether such early reactivations are functionally relevant to successful episodic retrieval. Theoretically, these early reactivation signatures could accompany memory retrieval, but they might not be causally relevant to the retrieval process. To investigate this question, we tested whether counteracting early sensory cortical activity with rTMS in the hemisphere contralateral to the site of encoding reduces retrieval of the episodic memory trace.

\section{Counteracting early sensory cortical activity through rTMS reduces episodic memory}

Behavioral task and procedures in Experiment 2 were identical to Experiment 1 (Fig. 1), except that, instead of measuring EEG, rTMS and Sham stimulation were applied during the retrieval phase of the experiment. Small breaks during the retrieval phases were used to relocate the TMS coil for the stimulation conditions (Sham, TMS) on the different stimulation sites, left hemisphere $(\mathrm{LH})$ or right hemisphere $(\mathrm{RH})$ in each subject. rTMS was applied at the average center EEG frequency observed during

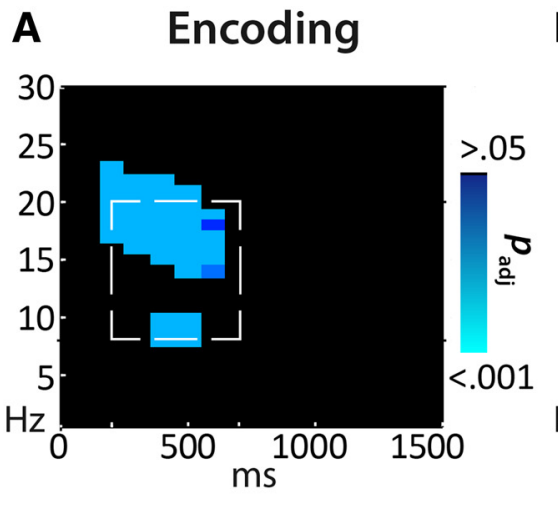

B Retrieval
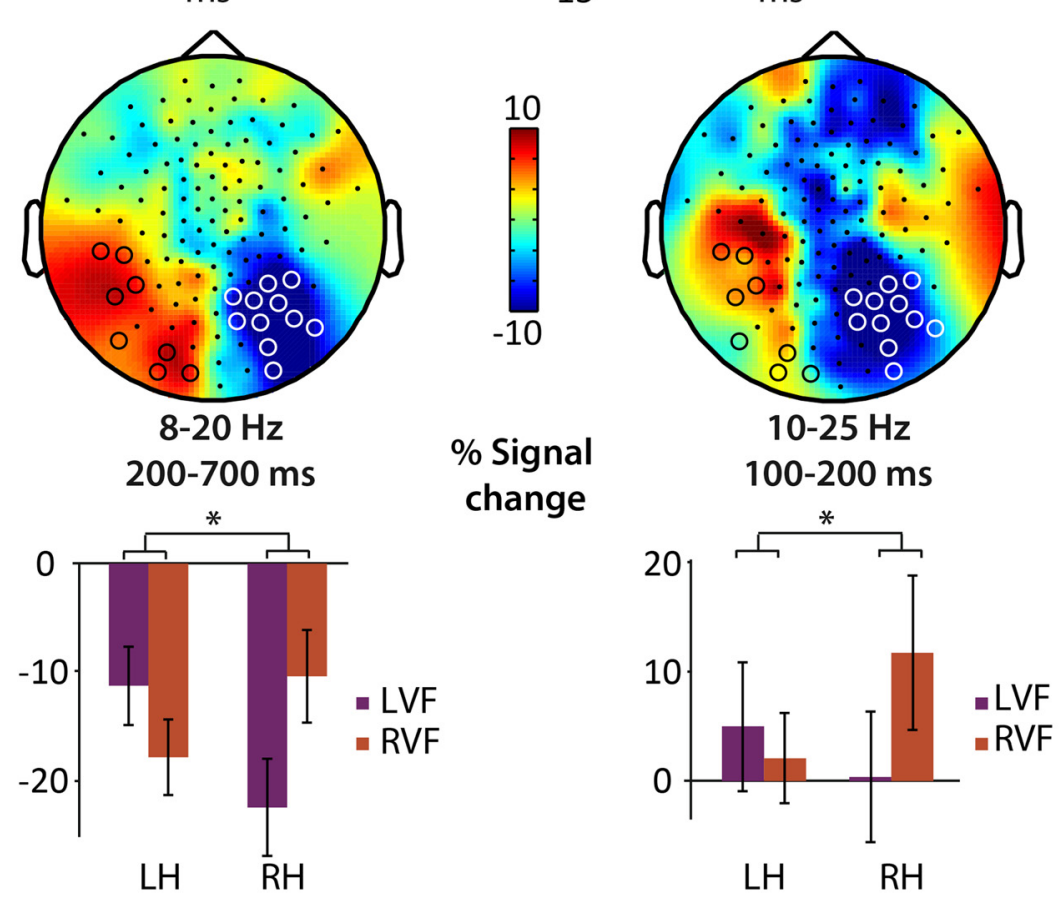

$\mathrm{LH} \quad \mathrm{RH}$

Figure 3. EEG activity in Experiment 1 at sensor level. $\boldsymbol{A}$, Encoding effects. Top, FDR-corrected results of the sliding cluster statistic, thresholded at $p_{\text {adj }}<0.05$, indicating significant power differences between LVF and RVF conditions at respective posterior contralateral sensors (compare Fig. 2) between $200-700 \mathrm{~ms}$ and $8-20 \mathrm{~Hz}$ (boxed white). Middle, LVF-RVF power differences in the selected time-frequency window. Significant electrode clusters interacting with VF condition represented by hemispheric (RH) electrode cluster interaction with VF condition (LVF and RVF). Error bars indicate SEM. *Significant interaction effect $(p<0.05)$. $\boldsymbol{B}$, Retrieval effects. Top, Mean difference between contralateral and ipsilateral EEG power for both VF conditions $10-25 \mathrm{~Hz}$, thresholded at $p<0.05$ (Wilcoxon sign rank test). The analysis focused on the time window preceding recollection effects ( $<500 \mathrm{~ms}$; postrecollection time window masked gray). Middle, LVF-RVF power differences in the selected time-frequency (left hemisphere) and white (right hemisphere) circles represent electrode clusters identified during encoding Bottom, Mean power at the left-hemispheric ( $(\mathrm{LH})$ and right-hemispheric $(\mathrm{RH})$ electrode clusters identified at encoding, interacting with VF condition (LVF and RVF). Error bars indicate SEM. *Significant interaction effect $(p<0.05)$.

retrieval $(17.5 \mathrm{~Hz})$ at the neural sources of the EEG retrieval effect obtained in Experiment 1 via a neuronavigation system (ANT-Visor; www.ant-neuro.com). rTMS was centered at the time interval of maximum EEG differences between 100 and $200 \mathrm{~ms}$. Driving neural assemblies in the LOC at $\alpha / \beta$ frequencies with 17.5 Hz rTMS (Thut et al., 2011; Hanslmayr et al., 2014) should counteract the decrease of $\alpha / \beta$ power observed in Experiment 1 and therefore impair episodic memory retrieval (Waldhauser et al., 2012).

As in Experiment 1, encoding condition (instructed vs noninstructed) had no effect on later item recognition or source memory performance, nor did visual field at encoding (all $t_{(23)}<$ 1.686, $p>0.05$; Table 1). Thus, behavioral data were again collapsed across these encoding conditions. In addition, we col- 
A

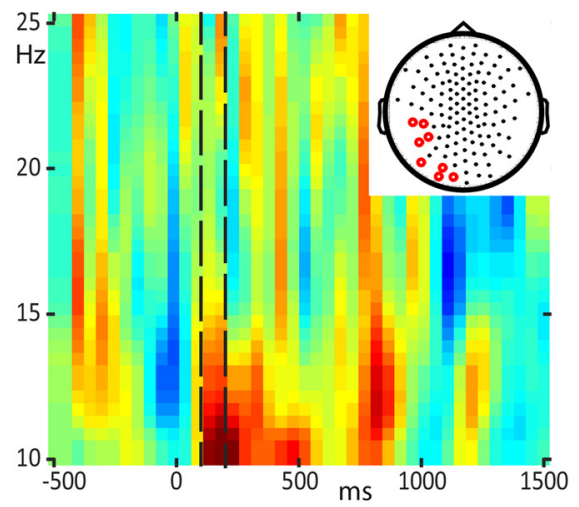

RH cluster

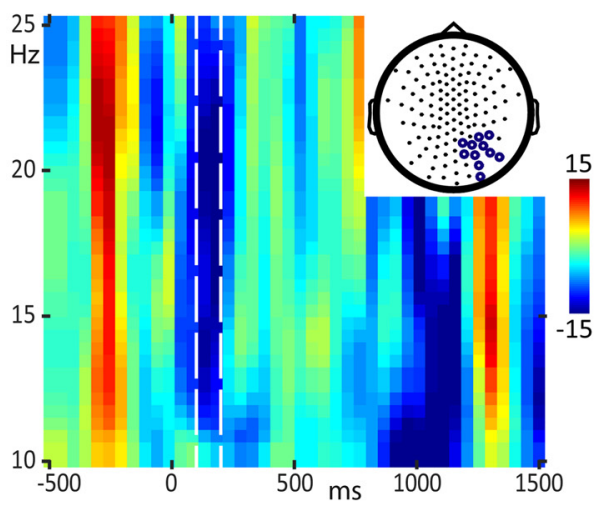

B

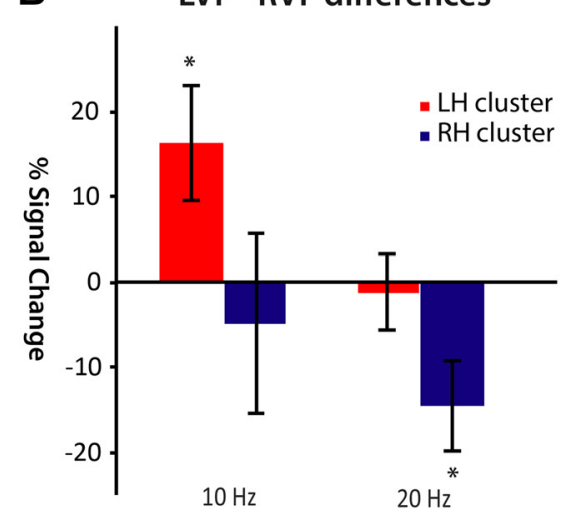

Figure 4. Hemisphere-specific effects for the LVF-RVF comparison. $\boldsymbol{A}$, Time-frequency representation of the LVF-RVF difference for left (red circles) and right (blue circles) hemispheric electrode clusters. The 100-200 ms time window selected on the basis of the running Wilcoxon test (Fig. 3B, top) is boxed black (LH) or white (RH). $\boldsymbol{B}$, Mean difference between LVF and RVF conditions at left and right hemispheric clusters between 100 and $200 \mathrm{~ms}$ at the hemisphere-specific peak frequencies ( $10 \mathrm{and} 20 \mathrm{~Hz}$ ). 0 nly the LH cluster shows a significant difference between VF conditions at 10 $\mathrm{Hz}$, whereas effects for the $\mathrm{RH}$ cluster are more pronounced at higher frequencies, peaking at $20 \mathrm{~Hz}$. Error bars indicate SEM. ${ }^{*}$ Significant difference $(p<0.05)$.

Table 2. Contingency between source memory performance and EEG lateralization in all single trials ${ }^{a}$

\begin{tabular}{lll}
\hline & $\mathrm{LI}<0$ & $\mathrm{LI}>0$ \\
\hline Source hits & $470(454)$ & $427(443)$ \\
Source misses & $123(139)$ & $151(135)$
\end{tabular}

${ }^{a}$ Contingency between lateralization index (LI) (contralateral - ipsilateral power) and source memory performance as observed absolute frequencies across all single trials of all subjects (expected values calculated in a $\chi^{2}$ test are given in parentheses). $\mathrm{LI}<0$ signifies an alpha/beta power decrease at the electrode cluster contralateral to the VF of encoding, as hypothesized for source hits, whereas $\mathrm{LI}>0$ signifies an ipsilateral decrease in the $100-200 \mathrm{~ms}$ time window.

\section{Source localization results}

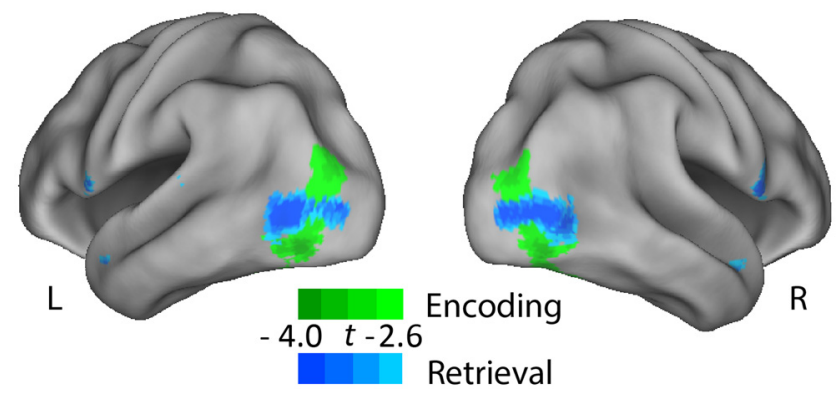

Figure 5. Cortical sources of EEG power differences between LVF and RVF conditions and between contralateral and ipsilateral hemispheres at encoding ( $8-20 \mathrm{~Hz}, 200-700 \mathrm{~ms}$; green) and retrieval (10-25 Hz, 100-200 ms; blue). Interhemispheric differences are backprojected to the cortical hemispheres, reflecting corresponding voxels in each hemisphere. Depicted $t$ values are thresholded at $p<$ 0.01 , with maximum values reflecting interhemispheric LVF-RVF differences at $p<0.0005$.

lapsed memory performance for items for which TMS was applied to the hemisphere contralateral versus ipsilateral to the VF of presentation during encoding. We assumed that rTMS should specifically decrease episodic memory performance for items that were presented contralateral to the hemisphere of stimulation. A two-way ANOVA on the percentage of correct source hits on all old items revealed a significant interaction between Stimulation (Sham vs rTMS) and Hemisphere (contralateral vs ipsilateral to VF of presentation; $F_{(1,23)}=4.617, p=0.042$; Table 3; Fig. $6 B$ ). No main effect for Stimulation or Hemisphere occurred in this analysis $\left(F_{(1,23)}<3.449, p>0.05\right)$. Source memory performance was significantly lower when rTMS was applied at the hemisphere contralateral to the VF of encoding compared with contralateral source memory performance in the Sham condition $\left(t_{(23)}=\right.$
Table 3. Memory performance depending on stimulation and hemisphere in Experiment $2^{a}$

\begin{tabular}{lrrrrr}
\hline & \multicolumn{1}{l}{ rTMS } & & \multicolumn{2}{l}{ Sham } \\
\cline { 2 - 3 } & \multicolumn{1}{c}{ Contralateral } & \multicolumn{1}{l}{ Ipsilateral } & & Contralateral & \multicolumn{1}{l}{ Ipsilateral } \\
\hline Source hits & $51.7 \pm 2.82$ & $54.6 \pm 2.61$ & & $58.8 \pm 3.22$ & $54.9 \pm 3.07$ \\
Hits & $69.2 \pm 2.61$ & $70.5 \pm 2.43$ & & $75.3 \pm 3.34$ & $70.3 \pm 3.15$ \\
Source FA & $4.0 \pm 0.55$ & $4.1 \pm 0.53$ & & $5.3 \pm 0.97$ & $3.4 \pm 0.52$ \\
\hline
\end{tabular}

${ }^{a}$ Percentages of hits, source hits, and source false alarms (mean \pm SEM) depending on stimulation (Sham vs rTMS) and hemisphere (Contralateral vs Ipsilateral).

2.410, $p=0.024$; Table 3; Fig. $6 B$ ). No difference between rTMS and Sham was found for ipsilateral stimulation $\left(t_{(23)}=0.160, p=\right.$ 0.874 , not significant; Table 3; Fig. $6 B$ ). Source hits did not differ between contralateral and ipsilateral hemispheres when analyzing rTMS and Sham stimulation data separately $\left(t_{(23)}<1.973\right.$, $p>0.05$; compare Table 3 ).

No main or interaction effects were obtained for item recognition as measured in hit rates, regardless of source memory performance $\left(F_{(1,23)}<2.474, p>0.05\right.$; Table 3$)$, suggesting that rTMS only had an influence on source memory but not item memory. However, there was no significant evidence $\left(F_{(1,23)}=\right.$ $0.013, p=0.911)$ that $r$ TMS affected source hits to a larger extent than hits as tested in a $2 \times 2 \times 2$ ANOVA with factors Type (source hits vs hits), Stimulation (Sham vs rTMS), and Hemisphere (contralateral vs ipsilateral), possibly because both measures are not fully independent from each other. To further explore the nature of the rTMS effect on memory, several control analyses were performed. For instance, we investigated in how far rTMS introduced a response bias. To this end, we tested whether rTMS increased false alarm rates and whether a potential effect would be dependent on the hemisphere of stimulation in a two-way repeated-measures ANOVA with factors Stimulation (rTMS, Sham) and Hemisphere (LH, RH). There was no significant main or interaction effect differentiating false alarm rates during $\mathrm{LH}$ (mean $\pm \mathrm{SD}, 9.5 \pm 9.00 \%)$ or $\mathrm{RH}(9.1 \pm 4.28 \%)$, rTMS and LH $(8.4 \pm 8.29 \%)$ or RH $(9.0 \pm 7.15 \%)$ Sham stimulation (all $\left.F_{(1,23)}<0.449, p>0.05\right)$. Second, we tested whether rTMS increased or decreased the tendency to attribute false alarms to the visual field contralateral to the site of stimulation (Table 3, source false alarms). Again, no significant main or interaction effect emerged in the two-way repeated-measures ANOVA with factors Stimulation (rTMS vs Sham) and Hemisphere (contralateral vs ipsilateral; all $F_{(1,23)}<3.206, p>0.05$ ). 


\section{A TMS stimulation}
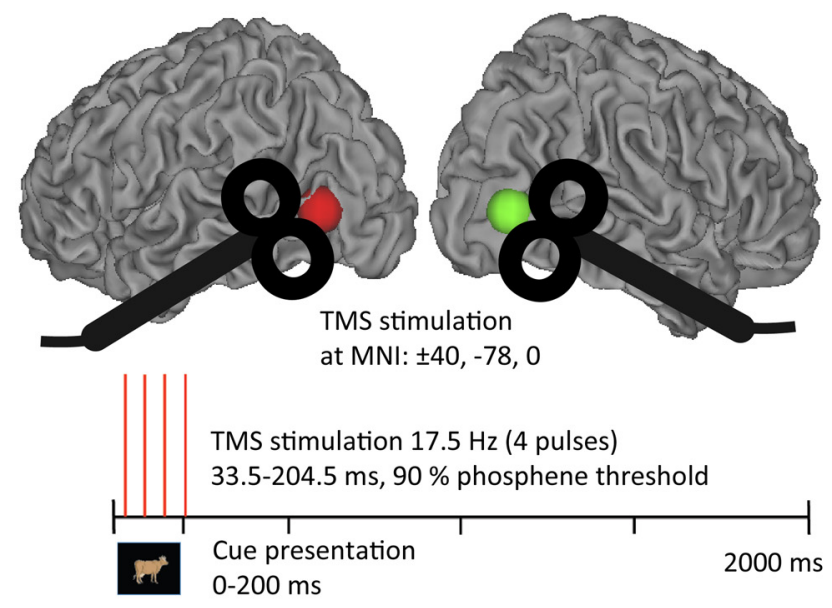

\section{B TMS results}

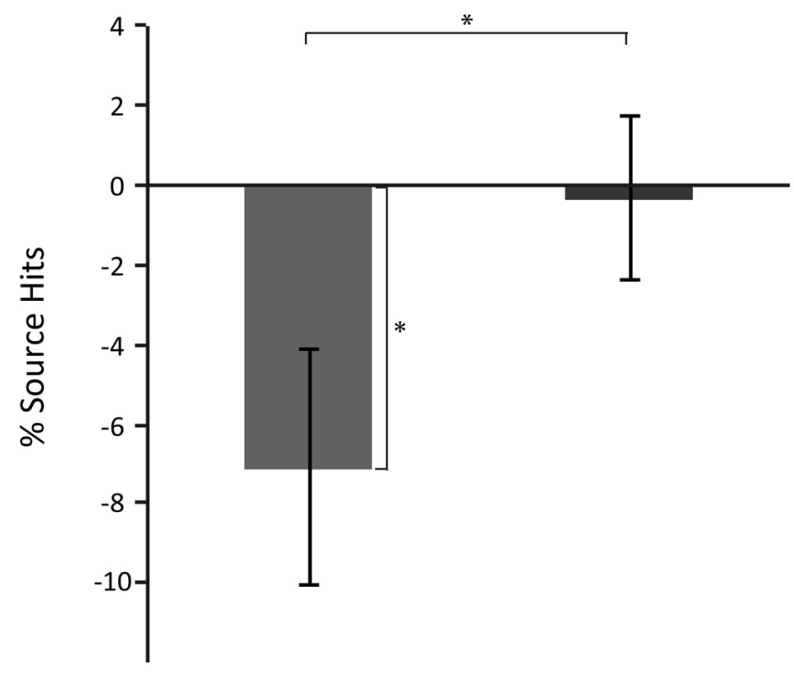

Contralatera

|psilateral

TMS stimulation - Sham

Figure 6. A, Schematic depiction of rTMS in the retrieval trial procedure of Experiment 2 at the left (red) and right (green) maximum cortical source (MNI coordinates: $\pm 40,-78,0$ ) of $10-25 \mathrm{~Hz}(100-200 \mathrm{~ms})$ interhemispheric LVF-RVF differences at retrieval as identified in Experiment 1. B, Behavioral results from Experiment 2, showing the difference between TMSSham condition effects on source memory performance for items presented in the contralateral and ipsilateral VF during encoding. Error bars indicate SEM. *Significant effect $(p<0.05)$.

Together, these analyses show that rTMS specifically affects source memory performance for items previously presented in the VF contralateral to the stimulated hemisphere compared with Sham stimulation. Such an effect could not be observed for hits regardless of source memory performance, but the reduction in source hit performance was not significantly different from performance for hits in general. Finally, we could rule out any unspecific effect of rTMS on response execution or memory bias by showing that Stimulation had no effect on the endorsement of new items in terms of false alarm rates. This suggests that $\alpha / \beta$ power decreases are especially relevant for episodic memory through providing rapid sensory reactivation as a basis for later source memory performance.

\section{Discussion}

We here show that very rapid reactivation of sensory information is functionally relevant for episodic memory retrieval. This conclusion is supported by the results of two independent experiments described above. First, lateralized encoding patterns of $\alpha / \beta$ power decreases reemerge rapidly in visual cortical areas during retrieval. Second, interfering with these early reactivation patterns reduces episodic memory retrieval. These findings add to the literature in demonstrating very rapid, context-specific memory reactivation, termed ecphory (Waldhauser et al., 2012; Wimber et al., 2012; Jafarpour et al., 2014; Johnson et al., 2015). Our results go one critical step beyond the previous findings in showing that these rapid reactivations of sensory memory traces are functionally relevant for episodic memory retrieval and directly affect the ability to retrieve contextual details of the study episode. These findings are a major conceptual advance for episodic memory research, providing first evidence that ecphoric processes are causally related to episodic memory retrieval.

Ecphory has long been suggested as a prerequisite for the "mental time travel" implied in retrieval from episodic memory (Tulving et al., 1983). It is assumed that incoming sensory information from retrieval cues reverberates with stored memory traces, leading to their immediate and involuntary reactivation (Tulving, 1982). While the theoretical idea has a long tradition in cognitive psychology (Semon, 1911) and is corroborated by behavioral data (Sheldon and Moscovitch, 2010), neural evidence for this process has been sparse. Typically, neural correlates of episodic memory retrieval were identified in a time range not before $\sim 300 \mathrm{~ms}$ after onset of a retrieval cue (Rugg and Curran, 2007). However, recent studies showed early reactivation patterns during episodic retrieval that are in line with our results (Waldhauser et al., 2012; Wimber et al., 2012; Jafarpour et al., 2014; Johnson et al., 2015). Albeit using different analysis strategies and stimuli, these studies show that a replay of context information can occur rapidly, well within $500 \mathrm{~ms}$ after presentation of a retrieval cue. The exact neural mechanisms of this sensory reactivation and its interaction with controlled retrieval processes are still unclear and deserve further investigation. According to cognitive theories, ecphory is a necessary but not sufficient prerequisite for episodic retrieval (Tulving, 1982, 1983; Moscovitch, 2008). The sensory information needs to be further processed by higher retrieval mechanisms, possibly through hippocampalneocortical loops (Horner et al., 2012; Staresina et al., 2012) and potentially guided by prefrontal and parietal control (Ranganath and Paller, 1999; Cabeza et al., 2008). As our data suggest, disrupting early sensory reactivation via external stimulation negatively affects the recollection (i.e., retrieval of contextual details) of episodic memories. Interestingly, our results match closely with one of our earlier studies that showed that inhibiting retrieval of interfering information leads to an increase in $\alpha / \beta$ oscillatory power in the hemisphere housing unwanted memory traces that interfere with the retrieval of a target memory trace (Waldhauser et al., 2012). This suggests that early modulations of $\alpha / \beta$ power are a decisive ingredient of successful remembering and can already act in concert with retrieval goals and be biased by higher-order cognitive control mechanisms.

Discussing the possible involvement of prefrontal control processes calls into question whether "ecphory" is the right label for the early sensory reactivation effects observed in the present data. However, as already mentioned in early papers on this topic, higher-order control mechanisms are likely to interact with rapid sensory reactivation during ecphory (Tulving et al., 1983; Johnson, 1992; Lepage et al., 2000; Rugg and Wilding, 2000). Recent neuroscientific studies also suggest that it is difficult to view these 
processes in separation, even at early stages of memory processing and during involuntary retrieval (Kompus, 2011; Kompus et al., 2011; Waldhauser et al., 2012).

In line with our hypothesis, a decrease of $\alpha / \beta$ oscillatory power at the hemisphere contralateral to the VF of encoding was most pronounced for source hits, to the extent that single trials could be classified as source hits or source misses on the basis of EEG lateralization. In the same vein, rTMS had a selective influence on source memory performance (albeit not to the extent of yielding significant differences between source hits, and hits; i.e., item memory). This pattern of results suggests that $\alpha / \beta$ power decreases are particularly relevant for source memory performance, which is in accordance with the theoretical notion of ecphory because correct source memory judgments require the highest amount of ecphoric information (Tulving et al., 1983). Unexpectedly, in the EEG analysis, source misses showed $\alpha / \beta$ lateralization in the opposite direction compared with source hits. Interpretation of this result has to remain speculative at this point. It appears that visual cortical activity predicted later memory decisions, such that a retrieved memory representation is attributed to the VF contralateral to the hemisphere where a decrease of $\alpha / \beta$ power occurred. It could be the case that memory representations attributed to the wrong VF were initially stored in the ipsilateral hemisphere during encoding. Alternatively, this misattribution may be due to nonsystematic fluctuations in $\alpha / \beta$ activity that led to illusory recollection of the wrong hemifield (e.g., Lange et al., 2014). Finally, it might be the case that EEG lateralization in general reflects a process of source reconstruction, and not sensory reactivation. However, this possibility seems rather unlikely because reconstructive processes during memory retrieval are more effortful and typically observed later $(>600 \mathrm{~ms})$ during retrieval processing (Johansson and Mecklinger, 2003; Herron, 2007). Future studies, possibly combining EEG and high-resolution fMRI or using electrophysiological methods that allow for assessing gamma oscillations (see below), might allow to distinguish between true versus false source memories in this paradigm (Slotnick and Schacter, 2004; Sederberg et al., 2007).

Our findings are in line with the idea that $\alpha / \beta$ power decreases reflect sensory information of episodic memory traces (Hanslmayr et al., 2012). However, we cannot conclude that these are the only frequency bands that are involved in ecphoric processing because we restrained analyses to low frequencies up to $30 \mathrm{~Hz}$ because EEG is not ideally suited to pick up the presumably very local high-frequency activities in the gamma range (Lopes da Silva, 2013). Furthermore, we restrained rTMS to the center frequency of the $\alpha / \beta$ range observed in Experiment 1 but did not apply stimulation with another frequency. Thus, although we have strong reason to assume that $\alpha / \beta$ power in visual cortex plays a decisive role for ecphoric processes, we cannot conclude that ecphory is specific to the $\alpha / \beta$ range. It might be that gamma, together with theta and alpha oscillations, also plays a decisive role in ecphoric processes (Osipova et al., 2006; Osipova et al., 2008). These are important questions that are beyond the scope of our study and should be addressed by future studies using techniques that allow for investigating these highfrequency activities (MEG or intracranial EEG) and applying different control frequencies in stimulation protocols (using TMS or transcranial alternating current stimulation). Another interesting question that is beyond the scope of our study is the role of hippocampal-neocortical interactions during early retrieval processes. For instance, does the rapid reactivation of sensory information depend on the hippocampus or is it a purely cortical or thalamocortical phenomenon (Staudigl et al., 2012; Headley and
Weinberger, 2015; Ketz et al., 2015)? Our results add an important angle to this research topic by suggesting that the earliest interactions between sensory information and stored memory traces may occur in the $\alpha / \beta$ oscillatory band, beyond the longdiscussed role of theta and gamma oscillations for memory processing. Finally, another open question is whether similar results would be observed in different sensory modalities or with different visual stimuli. Future studies, together with past research using different stimulus material and different experimental manipulations, are required to generalize our findings (Gratton et al., 1997; Wheeler and Buckner, 2003; Slotnick and Schacter, 2006; Waldhauser et al., 2012).

Together, our results show that retrieval from episodic memory leads to a very rapid reactivation of encoding activity, which is visible in $\alpha / \beta$ power decreases in visual brain regions. Affecting the cortical generators of the $\alpha / \beta$ power decrease with rTMS in the same frequency range hampered episodic memory retrieval. These observations suggest that episodic memory retrieval relies on ecphoric processes. A deeper understanding of ecphory and its manipulation with cortical stimulation techniques potentially offers new perspectives for the treatment of neuropsychiatric disorders (McNamara et al., 2001). For example, patients suffering from post-traumatic stress disorder suffer from the rapid intrusive reactivation of sensory memories pertaining to their traumatic experiences (Reynolds and Brewin, 1999). Treating memory intrusion through the external induction of oscillatory activity could be an important future therapeutic mean to assist patients in controlling unwanted memories.

\section{References}

Anderson AJ, Johnson CA (2006) Comparison of the ASA, MOBS, and ZEST threshold methods. Vision Res 46:2403-2411. CrossRef Medline

Batchelder WH, Riefer DM (1990) Multinomial processing models of source monitoring. Psychol Rev 97:548-564. CrossRef

Benjamini Y,Hochberg Y (1995) Controlling the false discovery rate: a practical and powerful approach to multiple testing. J R Stat Soc B 57:289-300.

Benjamini Y, Yekutieli D (2001) The control of the false discovery rate in multiple testing under dependency. Ann Stat 29:1165-1188. CrossRef

Cabeza R, Ciaramelli E, Olson IR, Moscovitch M (2008) The parietal cortex and episodic memory: an attentional account. Nat Rev Neurosci 9: 613-625. CrossRef Medline

Cycowicz YM, Friedman D, Snodgrass JG, Duff M (2001) Recognition and source memory for pictures in children and adults. Neuropsychologia 39:255-267. CrossRef Medline

Lopes da Silva FL (2013) EEG and MEG: relevance to neuroscience. Neuron 80:1112-1128. CrossRef Medline

Dalal SS, Guggisberg AG, Edwards E, Sekihara K, Findlay AM, Canolty RT, Berger MS, Knight RT, Barbaro NM, Kirsch HE, Nagarajan SS (2008) Fivedimensional neuroimaging: localization of the time-frequency dynamics of cortical activity. Neuroimage 40:1686-1700. CrossRef Medline

Genovese CR, Lazar NA, Nichols T (2002) Thresholding of statistical maps in functional neuroimaging using the false discovery rate. Neuroimage 15:870-878. CrossRef Medline

Gratton G (1998) The contralateral organization of visual memory: a theoretical concept and a research tool. Psychophysiology 35:638-647. CrossRef Medline

Gratton G, Corballis PM, Jain S (1997) Hemispheric organization of visual memories. J Cogn Neurosci 9:92-104. CrossRef Medline

Gross J, Kujala J, Hamalainen M, Timmermann L, Schnitzler A, Salmelin R (2001) Dynamic imaging of coherent sources: studying neural interactions in the human brain. Proc Natl Acad Sci U S A 98:694-699. CrossRef Medline

Gross J, Timmermann L, Kujala J, Salmelin R, Schnitzler A (2003) Properties of MEG tomographic maps obtained with spatial filtering. Neuroimage 19:1329-1336. CrossRef Medline

Händel BF, Haarmeier T, Jensen O (2011) Alpha oscillations correlate with the successful inhibition of unattended stimuli. J Cogn Neurosci 23: 2494-2502. CrossRef Medline

Hanslmayr S, Staudigl T, Fellner MC (2012) Oscillatory power decreases 
and long-term memory: the information via desynchronization hypothesis. Front Hum Neurosci 6:74. CrossRef Medline

Hanslmayr S, Matuschek J, Fellner MC (2014) Entrainment of prefrontal beta oscillations induces an endogenous echo and impairs memory formation. Curr Biol 24:904-909. CrossRef Medline

Headley DB, Weinberger NM (2015) Relational associative learning induces crossmodal plasticity in early visual cortex. Cereb Cortex 25:1306-1318. CrossRef Medline

Herron JE (2007) Decomposition of the ERP late posterior negativity: effects of retrieval and response fluency. Psychophysiology 44:233-244. CrossRef Medline

Horner AJ, Gadian DG, Fuentemilla L, Jentschke S, Vargha-Khadem F, Duzel E (2012) A rapid, hippocampus-dependent, item-memory signal that initiates context memory in humans. Curr Biol 22:2369-2374. CrossRef Medline

Jafarpour A, Fuentemilla L, Horner AJ, Penny W, Duzel E (2014) Replay of very early encoding representations during recollection. J Neurosci 34 : 242-248. CrossRef Medline

Johansson M, Mecklinger A (2003) The late posterior negativity in ERP studies of episodic memory: action monitoring and retrieval of attribute conjunctions. Biol Psychol 64:91-117. CrossRef Medline

Johnson JD, Price MH, Leiker EK (2015) Episodic retrieval involves early and sustained effects of reactivating information from encoding. Neuroimage 106:300-310. CrossRef Medline

Johnson MK (1992) MEM: mechanisms of recollection. J Cogn Neurosci 4:268-280. CrossRef Medline

Ketz NA, Jensen O, O’Reilly RC (2015) Thalamic pathways underlying prefrontal cortex-medial temporal lobe oscillatory interactions. Trends Neurosci 38:3-12. CrossRef Medline

Kompus K (2011) Default mode network gates the retrieval of task-irrelevant incidental memories. Neurosci Lett 487:318-321. CrossRef Medline

Kompus K, Eichele T, Hugdahl K, Nyberg L (2011) Multimodal imaging of incidental retrieval: the low route to memory. J Cogn Neurosci 23:947960. CrossRef Medline

Konen CS, Kastner S (2008) Two hierarchically organized neural systems for object information in human visual cortex. Nat Neurosci 11:224-231. CrossRef Medline

Lange J, Keil J, Schnitzler A, van Dijk H, Weisz N (2014) The role of alpha oscillations for illusory perception. Behav Brain Res 271:294-301. CrossRef Medline

Lepage M, Ghaffar O, Nyberg L, Tulving E (2000) Prefrontal cortex and episodic memory retrieval mode. Proc Natl Acad Sci U S A 97:506-511. CrossRef Medline

Maris E, Oostenveld R (2007) Nonparametric statistical testing of EEG- and MEG-data. J Neurosci Methods 164:177-190. CrossRef Medline

McNamara B, Ray JL, Arthurs OJ, Boniface S (2001) Transcranial magnetic stimulation for depression and other psychiatric disorders. Psychol Med 31:1141-1146. Medline

Moscovitch M (2008) The hippocampus as a "stupid," domain-specific module: implications for theories of recent and remote memory, and of imagination. Can J Psychol 62:62-79. CrossRef Medline

Oldfield RC (1971) The assessment and analysis of handedness: the Edinburgh Inventory. Neuropsychologia 9:97-113. CrossRef Medline

Oostenveld R, Fries P, Maris E, Schoffelen JM (2011) FieldTrip: open source software for advanced analysis of MEG, EEG, and invasive electrophysiological data. Comput Intell Neurosci 2011:156869. CrossRef Medline

Osipova D, Takashima A, Oostenveld R, Fernández G, Maris E, Jensen O (2006) Theta and gamma oscillations predict encoding and retrieval of declarative memory. J Neurosci 26:7523-7531. CrossRef Medline

Osipova D, Hermes D, Jensen O (2008) Gamma power is phase-locked to posterior alpha activity. PLoS One 3:e3990. CrossRef Medline

Ranganath C, Paller KA (1999) Frontal brain potentials during recognition are modulated by requirements to retrieve perceptual detail. Neuron 22: 605-613. CrossRef Medline

Reynolds M, Brewin CR (1999) Intrusive memories in depression and posttraumatic stress disorder. Behav Res Ther 37:201-215. CrossRef Medline

Romei V, Gross J, Thut G (2010) On the role of prestimulus alpha rhythms over occipito-parietal areas in visual input regulation: correlation or causation? J Neurosci 30:8692-8697. CrossRef Medline

Rossi S, Hallett M, Rossini PM, Pascual-Leone A (2009) Safety, ethical con- siderations, and application guidelines for the use of transcranial magnetic stimulation in clinical practice and research. Clin Neurophysiol 120:2008-2039. CrossRef Medline

Rossi S, Hallett M, Rossini PM, Pascual-Leone A (2011) Screening questionnaire before TMS: an update. Clin Neurophysiol 122:1686. CrossRef Medline

Rossion B, Pourtois G (2004) Revisiting Snodgrass and Vanderwart's object pictorial set: the role of surface detail in basic-level object recognition. Perception 33:217-236. CrossRef Medline

Rugg MD, Curran T (2007) Event-related potentials and recognition memory. Trends Cogn Sci 11:251-257. CrossRef Medline

Rugg MD, Wilding EL (2000) Retrieval processing and episodic memory. Trends Cogn Sci 4:108-115. CrossRef Medline

Sauseng P, Klimesch W, Heise KF, Gruber WR, Holz E, Karim AA, Glennon M, Gerloff C, Birbaumer N, Hummel FC (2009) Brain oscillatory substrates of visual short-term memory capacity. Curr Biol 19:1846-1852. CrossRef Medline

Sederberg PB, Schulze-Bonhage A, Madsen JR, Bromfield EB, Litt B, Brandt A, Kahana MJ (2007) Gamma oscillations distinguish true from false memories. Psychol Sci 18:927-932. CrossRef Medline

Semon RW (1911) Die Mneme als erhaltendes Prinzip im Wechsel des organischen Geschehens. Leipzig, Germany: Engelmann.

Sheldon SA, Moscovitch M (2010) Recollective performance advantages for implicit memory tasks. Memory 18:681-697. CrossRef Medline

Slotnick SD (2004) Visual memory and visual perception recruit common neural substrates. Behav Cogn Neurosci Rev 3:207-221. CrossRef Medline

Slotnick SD, Schacter DL (2004) A sensory signature that distinguishes true from false memories. Nat Neurosci 7:664-672. CrossRef Medline

Slotnick SD, Schacter DL (2006) The nature of memory related activity in early visual areas. Neuropsychologia 44:2874-2886. CrossRef Medline

Slotnick SD, Schacter DL (2010) Conscious and nonconscious memory effects are temporally dissociable. Cogn Neurosci 1:8-15. CrossRef Medline

Staresina BP, Fell J, Do Lam AT, Axmacher N, Henson RN (2012) Memory signals are temporally dissociated in and across human hippocampus and perirhinal cortex. Nat Neurosci 15:1167-1173. CrossRef Medline

Staudigl T, Hanslmayr S (2013) Theta oscillations at encoding mediate the context-dependent nature of human episodic memory. Curr Biol 23: 1101-1106. CrossRef Medline

Staudigl T, Zaehle T, Voges J, Hanslmayr S, Esslinger C, Hinrichs H, Schmitt FC, Heinze HJ, Richardson-Klavehn A (2012) Memory signals from the thalamus: early thalamocortical phase synchronization entrains gamma oscillations during long-term memory retrieval. Neuropsychologia 50: 3519-3527. CrossRef Medline

Thakral PP, Slotnick SD, Schacter DL (2013) Conscious processing during retrieval can occur in early and late visual regions. Neuropsychologia 51:482-487. CrossRef Medline

Thut G, Veniero D, Romei V, Miniussi C, Schyns P, Gross J (2011) Rhythmic TMS causes local entrainment of natural oscillatory signatures. Curr Biol 21:1176-1185. CrossRef Medline

Tulving E (1976) Ecphoric processes in recall and recognition. In: Recall and recognition (Brown J, ed), pp 37-73. Oxford: Wiley.

Tulving E (1982) Synergistic ecphory in recall and recognition. Can J Psychol 36:130-147. CrossRef

Tulving E (1983) Elements of episodic memory. Oxford: Oxford UP.

Tulving E, Le Voi ME, Routh DA, Loftus E (1983) Ecphoric processes in episodic memory [and discussion]. Philos Trans R Soc B 302:361-371. CrossRef

Tyrrell RA, Owens DA (1988) A rapid technique to assess the resting states of the eyes and other threshold phenomena: the modified binary search (MOBS). Behav Res Meth Ins 20:137-141. CrossRef

Waldhauser GT, Johansson M, Hanslmayr S (2012) $\alpha / \beta$ oscillations indicate inhibition of interfering visual memories. J Neurosci 32:1953-1961. CrossRef Medline

Wheeler ME, Buckner RL (2003) Functional dissociation among components of remembering: control, perceived oldness, and content. J Neurosci 23:3869-3880. Medline

Wimber M, Maa $\beta$ A, Staudigl T, Richardson-Klavehn A, Hanslmayr S (2012) Rapid memory reactivation revealed by oscillatory entrainment. Curr Biol 22:1482-1486. CrossRef Medline 